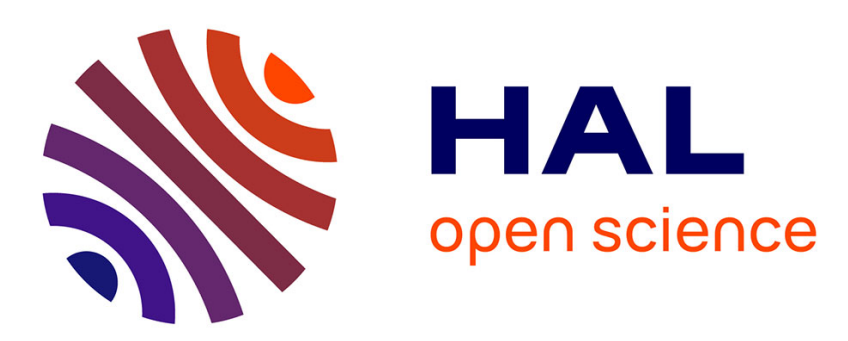

\title{
Questions ouvertes sur les effets des ondes électromagnétiques et sonores sur la santé et l'environnement
}

Olivier Merckel

\section{- To cite this version:}

Olivier Merckel. Questions ouvertes sur les effets des ondes électromagnétiques et sonores sur la santé et l'environnement. Annales des mines - Série Responsabilité et environnement, 2021, 103 (3), pp.29-32. 10.3917/re1.103.0029 . anses-03279000

\section{HAL Id: anses-03279000}

\section{https://hal-anses.archives-ouvertes.fr/anses-03279000}

Submitted on 6 Jul 2021

HAL is a multi-disciplinary open access archive for the deposit and dissemination of scientific research documents, whether they are published or not. The documents may come from teaching and research institutions in France or abroad, or from public or private research centers.
L'archive ouverte pluridisciplinaire HAL, est destinée au dépôt et à la diffusion de documents scientifiques de niveau recherche, publiés ou non, émanant des établissements d'enseignement et de recherche français ou étrangers, des laboratoires publics ou privés. 


\title{
Questions ouvertes sur les effets des ondes électromagnétiques et sonores sur la santé et l'environnement
}

\author{
Par Olivier MERCKEL
}

Agence nationale de sécurité sanitaire de l'alimentation, de l'environnement et du travail (Anses)

\begin{abstract}
Les spectres électromagnétiques et sonores sont loin de constituer des entités homogènes, notamment en matière d'interactions entre les ondes et les êtres vivants. Si certaines gammes de fréquences font l'objet de recherches soutenues en matière d'effets éventuels sur la santé, par exemple dans les domaines des communications mobiles ou des fréquences audibles, d'autres restent largement inexplorées. Et, même pour les plus étudiées, l'accumulation des connaissances, qui ne met pas en évidence, à l'heure actuelle, d'impact majeur en matière de santé publique, laisse ouvertes un certain nombre de questions, par exemple concernant des hypothèses de mécanismes d'action liés aux modulations des signaux, à leur fréquence, à la possibilité d'effets sur l'activité cérébrale ou encore à des symptômes associés par certaines personnes à leur exposition aux ondes électromagnétiques ou acoustiques. L'effet de ces ondes sur l'environnement est par ailleurs, aujourd'hui encore, pratiquement inexploré.
\end{abstract}

\section{Introduction}

Alors qu'il n'a pas été démontré à ce jour d'impact majeur de l'exposition aux ondes électromagnétiques en matière de santé publique, la production d'articles consacrés à la recherche d'effets éventuels reste soutenue. Des questions restent en effet toujours ouvertes, concernant par exemple des hypothèses de mécanismes d'interactions au niveau cellulaire, en particulier les échanges thermiques de faible intensité et leurs répercussions possibles, à court, moyen ou long termes, sur les organes ou le métabolisme chez l'animal.

Les effets des ondes acoustiques d'intensité élevée sur l'audition sont bien connus, mais les mécanismes sousjacents aux impacts du bruit sur la santé, par ailleurs de mieux en mieux caractérisés, sont encore mal compris.

Entre les interactions bien décrites des ondes de forte intensité avec le vivant et certaines affirmations non validées, il existe un espace pour des questions encore ouvertes concernant les effets éventuels des ondes électromagnétiques et sonores sur la santé et l'environnement.

\section{Entre consensus et incertitudes}

Selon que l'on adopte le point de vue de chercheurs, d'organismes d'expertise, d'associations militantes ou encore de particuliers, l'état des connaissances concernant les effets sur la santé de l'exposition aux champs et aux ondes électromagnétiques non ionisants (1), ainsi qu'aux ondes sonores, peut apparaître aussi bien sans relief que particulièrement contrasté. Dans un domaine pourtant largement investi par la recherche d'effets potentiels sur le vivant, la controverse est encore active, y compris sur la qualification et la quantification des risques, présents et à venir, parfois même au sein de la communauté scientifique. Si des interrogations concernant les effets éventuels sur la santé des ondes électromagnétiques ou sonores trouvent assez naturellement leur origine dans l'émergence de nouvelles modalités d'exposition (déploiement de nouvelles technologies de communication mobile - la 5G, par exemple -, évolutions technologiques - compteur Linky -, ou encore le développement de parcs éoliens) ; d'autres persistent, à bas bruit, depuis de nombreuses années au gré, par exemple, d'aménagements locaux du territoire, en lien notamment avec les lignes de transport d'électricité à haute tension.

Le consensus scientifique, partagé et accepté par les acteurs mobilisés sur la controverse " ondes et santé ", se réduit à la reconnaissance, d'une part, de la stimulation du système nerveux par les champs magnétiques basses

(1) On parlera plus volontiers de " champs électromagnétiques " pour désigner les composantes non propagatives des rayonnements émis par des sources électromagnétiques, alors que le terme « onde » fait référence, en règle générale, à la propagation de l'énergie électromagnétique à partir d'une source. 
fréquences, et de ce qui est désigné sous les termes d'effets thermiques des ondes électromagnétiques radiofréquences et, d'autre part, des effets auditifs des ondes acoustiques. Dans un cas comme dans l'autre, c'est l'exposition à des intensités très élevées de champs ou ondes électromagnétiques, ou acoustiques, qui est responsable des effets observés. Des intensités très élevées qui ne sont pas rencontrées, sauf cas accidentel, dans les activités de la population générale, mais peuvent être subies dans le cadre de l'exercice de certaines professions, qui adoptent donc des mesures de prévention pour s'en protéger.

En dehors de ces situations, différentes visions s'affrontent encore aujourd'hui, y compris dans une partie de la communauté scientifique, sur l'existence ou non d'effets délétères pour la santé de l'exposition aux ondes électromagnétiques ou acoustiques à des niveaux inférieurs aux valeurs limites réglementaires. Les effets biologiques observés à la suite d'une exposition à un agent externe (ici les ondes électromagnétiques ou acoustiques) peuvent être définis comme des changements d'ordre biochimique ou physiologique, ou du comportement, qui interviennent dans une cellule, un organe ou encore l'organisme complet. En permanence, les êtres vivants sont exposés à des stimulations internes et externes, et ces effets biologiques marquent les réponses adaptatives normales des cellules, des organes ou des organismes à ces stimulations. Un effet sur la santé d'un individu n'intervient que lorsque les effets biologiques (quelle que soit leur cause) dépassent les limites d'adaptation de l'organisme. Bien entendu, il est important de considérer les effets éventuels sur la santé qui peuvent s'observer à long terme, longtemps après des expositions qui n'auraient engendré, sur le moment, que des effets biologiques, ou à la suite d'expositions chroniques de faible intensité, par exemple.

Les champs et ondes électromagnétiques transportent de l'énergie électromagnétique. Cette énergie interagit avec la matière vivante selon différentes modalités, en particulier en fonction de la fréquence des rayonnements. Aux fréquences basses, l'énergie est plutôt convertie en courants électriques, qui peuvent interagir, par exemple, avec le système nerveux. Aux fréquences plus élevées, à partir de $100 \mathrm{kHz}$ environ, l'énergie déposée est transformée en énergie mécanique, au niveau moléculaire, puis thermique, par un mécanisme bien décrit (Foster et Schwan, 1996), avant que des processus de dissipation n'interviennent, par conduction dans les tissus et via la circulation sanguine (Foster et Glaser, 2007). Tant que la quantité d'énergie convertie est faible par rapport aux capacités d'adaptation de l'organisme (thermorégulation), aucun effet macroscopique local ou systémique n'est observé.

Par ailleurs, il n'est pas exclu, bien qu'aucun mécanisme n'ait été encore démontré, que l'apport d'énergie électromagnétique puisse provoquer des effets biologiques autres qu'un effet thermique, soit directement, soit indirectement (Pelletier, 2013)

Les incertitudes, ou plus précisément les questions encore ouvertes en matière d'effets éventuels de l'exposition aux ondes sur la santé animale et humaine, se situent également dans ces liens, pour l'heure hypothétiques, entre effets biologiques et effets sanitaires. Jusque dans les années 2010, une grande partie de la communauté scientifique s'intéressant aux effets des ondes électromagnétiques ou acoustiques sur le vivant tenait pour acquis qu'en dehors d'expositions à des niveaux très élevés, aucun effet, même biologique, n'était observé. La publication de travaux de recherche récents, à la fois dans le domaine des ondes électromagnétiques et celui des ondes acoustiques, ont quelque peu fait évoluer cette vision. En effet, si des incertitudes demeurent concernant, par exemple, des effets sur l'ADN ou le fonctionnement cellulaire, dont il est difficile souvent de savoir s'il s'agit de fluctuations biologiques endogènes ou d'un effet de l'exposition aux ondes, les données semblent plus robustes notamment pour celles concernant l'effet des ondes électromagnétiques sur le fonctionnement cérébral.

\section{Des questions ouvertes}

Un impact des ondes électromagnétiques sur le fonctionnement cérébral ?

En raison de l'utilisation des téléphones mobiles au contact de la tête, l'impact éventuel des radiofréquences sur le fonctionnement cérébral, son activité électrique, mais également les fonctions cognitives ou le sommeil a été exploré, de façon plus ou moins complète. Par fonction cognitive, on entend en particulier l'attention, la mémoire ainsi que les fonctions liées au langage, aux gestes ou encore à la coordination. En étudiant, par exemple, des paramètres d'enregistrements encéphalographiques chez l'homme, des modifications peuvent être observées sous une exposition aux radiofréquences (Croft et al., 2010). Quelques études chez l'animal laissent par ailleurs penser que l'exposition aux ondes électromagnétiques émises par des téléphones mobiles pourrait modifier les performances cognitives et la mémoire, voire de façon a priori étonnante, les améliorer (Kumlin et al., 2007 ; Arendash et al., 2010). Chez l'homme, et en particulier chez les enfants, quelques expériences méritent de leur accorder notre attention : des études rapportent des modifications de paramètres d'encéphalogrammes, lors d'une exposition en aveugle aux radiofréquences, pour les ondes émises par le cerveau correspondant à l'attention, à la mémoire, ainsi qu'aux émotions et aux sensations (Krause et al., 2006 ; Loughran et al., 2013). On peut également rapprocher ces observations de celles obtenues dans le domaine des basses fréquences, pour lesquelles on retrouve une interférence possible avec l'activité électrique cérébrale (voir, par exemple, Carrubba et al., 2010). À ce jour, cependant, aucune incidence pathologique de ces observations n'a été mise en évidence.

\section{Des effets spécifiques des signaux?}

Une des questions encore ouvertes concernant les effets possibles des ondes électromagnétiques sur la santé est relative à la distinction entre, d'une part, ceux qui seraient liés à l'énergie véhiculée par les rayonnements, indépendamment de la répartition temporelle avec laquelle cette énergie est transmise aux structures biologiques et, d'autre part, ceux qui seraient liés précisément à la structure temporelle des émissions, autrement dit à la 
modulation dans le temps des signaux. Les résultats des études menées sur des signaux dont les caractéristiques de modulation temporelle sont différentes, sont souvent disparates : des effets sur le fonctionnement biologique cellulaire peuvent être, par exemple, retrouvés pour des expositions à des signaux GSM ${ }^{(2)}$, mais pas en UMTS ${ }^{(3)}$, et réciproquement (voir, par exemple, Tillmann, Ernst et al., 2010 ; Danker-Hopfe et al., 2011 ; Zeni et al., 2012 ; Smith-Roe et al., 2019). Par ailleurs, l'hypothèse de démodulation des signaux qui pourrait être réalisée par des structures biologiques a fait l'objet d'études spécifiques (voir, par exemple, Sheppard et al., 2008 ; Davis et al., 2010 ; Kowalczuk et al., 2010). Ces études n'ont pas mis en évidence une capacité des entités biologiques, à petite ou à grande échelle, à démoduler les signaux, et donc à potentiellement réagir à la forme temporelle du signal plutôt qu'à l'énergie véhiculée. À l'exception de l'étude de Carubba et al. (2010), dont les auteurs avancent la possibilité d'émission d'un potentiel évoqué cérébral lié à l'exposition à un signal basse fréquence de $217 \mathrm{~Hz}$. Les études récentes sur les membranes cellulaires, naturelles ou artificielles, proposent d'intéressantes pistes de mécanismes d'action des champs électromagnétiques, notamment dans la gamme des ondes dites « millimétriques » (celles au-dessus de $10 \mathrm{GHz}$ ).

\section{Des effets liés aux fréquences ?}

Par définition, le spectre des fréquences, qu'elles soient sonores ou électromagnétiques, comporte une infinité de valeurs. Il est donc illusoire de penser pouvoir évaluer tous les risques éventuels liés à l'ensemble des fréquences. En dehors des phénomènes de résonance, qui sont importants à prendre en compte, et qui concernent donc des fréquences précises, les interactions entre les ondes et le vivant sont dirigées, notamment, par des mécanismes physiques liés, d'une part, aux rapports de grandeur entre les longueurs d'ondes et les objets illuminés et, d'autre part, à la nature de la matière en interaction. Les modes d'interaction entre les ondes et le vivant peuvent donc être définis dans des bandes de fréquences plus ou moins étendues, mais la question de savoir dans quelle largeur de bande de fréquences il est possible d'envisager que d'éventuels effets biologiques de même nature sont observables, reste ouverte. À l'heure actuelle, l'examen des résultats de plusieurs études publiées, qui ont exposé des cultures cellulaires ou des organismes entiers, par exemple, à des fréquences différentes, dans la bande $800 \mathrm{MHz}$ à $2,5 \mathrm{GHz}$, ne permet pas de conclure si la fréquence joue un rôle ou non dans la nature ou l'intensité des effets observés (Anses, 2021). On remarquera toutefois une plage étroite de fréquences, autour de 6 à $10 \mathrm{GHz}$, dans laquelle la modification des interactions entre ondes électromagnétiques et tissus biologiques est très rapide, et qui voit l'interface air-peau devenir, au-dessus de $10 \mathrm{GHz}$, une « barrière » à la fois réfléchissante et absorbante. Les

(2) GSM : Global System for Mobile Communications - La deuxième génération de téléphonie mobile.

(3) UMTS : Universal Mobile Telecommunications Systems - La troisième génération de téléphonie mobile. effets éventuels de résonance de ces ondes sur les organismes de petite taille (insectes...) n'a quasiment fait l'objet d'aucune étude.

« Intolérances idiopathiques environnementales » ou effets cumulés ?

Hormis les effets liés à l'échauffement des tissus et certains effets sur l'activité électrique cérébrale, notamment, l'exposition aux radiofréquences est-elle susceptible d'induire des symptômes à court ou moyen termes, tels que ceux décrits par les personnes qui se déclarent électrohypersensibles ? Aucune preuve d'une causalité entre l'exposition aux champs électromagnétiques et la description de ces symptômes n'a pu être apportée jusqu'ici (Anses, 2018). Les effets cumulés de ces expositions physiques entre elles, ou avec des expositions chimiques, n'ont pas fait l'objet de publications à ce jour. Ces symptômes (fatigue, maux de tête, vertiges, douleurs...) sont également décrits par des personnes qui les associent à leur exposition aux bruits, notamment aux infrasons produits par des éoliennes (Anses, 2017), ou qui sont exposées à des polluants chimiques. Ces symptômes sont parfois regroupés dans la littérature scientifique sous l'expression d'« intolérance idiopathique environnementale », laquelle associe ces symptômes aux champs électromagnétiques ou aux éoliennes, par exemple.

\section{Conclusion}

Avec l'exposition aux ondes électromagnétiques qui ne cesse de croître dans un quotidien toujours plus connecté, la recherche de leurs effets éventuels reste nécessaire, en particulier pour tenter de répondre aux questions toujours ouvertes, concernant par exemple leurs effets sur le fonctionnement cérébral, ou l'impact éventuel de nouvelles fréquences appelées à être massivement utilisées. Mais la structuration de la recherche dans ce domaine est-elle adaptée pour trouver des réponses à toutes ces interrogations ? Dans ce qui peut apparaître comme un mouvement brownien, compter sur un effet vertueux du foisonnement des thèmes d'étude et sur la sérendipité pour espérer des découvertes fondamentales pourrait être un écueil pour la santé publique. En effet, l'évaluation des risques est fondée sur l'analyse des données produites, qui restent manifestement hétérogènes malgré les agendas de recherche publiés régulièrement par l'Organisation mondiale de la santé ou par l'Anses, en France, dans le cadre de son appel annuel à projets de recherche.

En nous affranchissant de tout a priori, une question ouverte semble également très peu explorée : celle des effets éventuels des ondes électromagnétiques et acoustiques sur l'environnement. Alors que la santé des populations humaines est, dans certains domaines, de mieux en mieux suivie, celle des mondes animal et végétal semble, en dehors des espèces utilisées en agroalimentaire, bien peu observée. Les interactions de ces ondes avec notre environnement, si elles existent, pourraient constituer un sujet d'étude à la profondeur insoupçonnée. 


\section{Bibliographie}

ANSES (2017), " Évaluation des effets sanitaires des basses fréquences sonores et infrasons dus aux parcs éoliens ».

ANSES (2018), " Hypersensibilité électromagnétique ou intolérance environnementale idiopathique attribuées aux champs électromagnétiques ".

ANSES (2021), "Expositions aux champs électromagnétiques liées au déploiement de la technologie de communication " $5 \mathrm{G}$ " et effets sanitaires éventuels associés ".

ARENDASH G. W., SANCHEZ-RAMOS J. et al. (2010), "Electromagnetic field treatment protects against and reverses cognitive impairment in Alzheimer's disease mice", Journal of Alzheimer's Disease 19(1), pp. 191-210.

CARRUBBA S., FRILOT II C., CHESSON JR A. L. \& MARINO A. A. (2010), "Mobile-phone pulse triggers evoked potentials", Neuroscience Letters 469(1), pp. 164-168.

CROFT R. J., LEUNG S. et al. (2010), "Effects of $2 \mathrm{G}$ and $3 G$ mobile phones on human alpha rhythms: Resting EEG in adolescents, young adults, and the elderly", Bioelectromagnetics 31(6), pp. 434-444

DANKER-HOPFE H., DORN H. et al. (2011), "Effects of electromagnetic fields emitted by mobile phones (GSM 900 and WCDMA/UMTS) on the macrostructure of sleep", Journal of Sleep Research 20 (1 PART I), pp. 73-81.

DAVIS C. C. \& BALZANO Q. (2010), "The brain is not a radio receiver for wireless phone signals: Human tissue does not demodulate a modulated radiofrequency carrier", Comptes Rendus Physique 11 (9-10), pp. 585-591.

FOSTER K. R. \& SCHWAN H. P. (1996), "Dielectric properties of tissues", Handbook of biological effects of electromagnetic fields, CRC Press, pp. 25-102.

FOSTER, K. R. \& GLASER R. (2007), "Thermal mechanisms of interaction of radiofrequency energy with biological systems with relevance to exposure guidelines", Health Physics 92 (6), pp. 609620.
KOWALCZUK C. et al. (2010), "Absence of nonlinear responses in cells and tissues", Bioelectromagnetics 31(7), pp. 556-565.

KRAUSE C. M. et al. (2006), "Mobile phone effects on children's event-related oscillatory EEG during an auditory memory task", International Journal of Radiation Biology 82, pp. 443-450.

KUMLIN T., IIVONEN H. et al. (2007), "Mobile phone radiation and the developing brain: Behavioral and morphological effects in juvenile rats", Radiation Research 168(4), pp. 471-479.

LOUGHRAN S. P. et al. (2013), "No increased sensitivity in brain activity of adolescents exposed to mobile phone-like emissions", Clinical Neurophysiology 124, pp. 1303-1308.

PELLETIER A. et al. (2013), "Effects of chronic exposure to radiofrequency electromagnetic fields on energy balance in developing rats", Environmental Science \& Pollution Research - International 20(5), pp. 2735-2746.

SHEPPARD A. R. et al. (2008), "Quantitative evaluations of mechanisms of radiofrequency interactions with biological molecules and processes", Health Physics 95(4), pp. 365-396.

SMITH-ROE S. L. et al. (2019), "Evaluation of the genotoxicity of cell phone radiofrequency radiation in male and female rats and mice following subchronic exposure", Environmental and Molecular Mutagenenesis, octobre, doi: 10.1002/em.22343.

TILLMANN T., ERNST H. et al. (2010), "Indication of cocarcinogenic potential of chronic UMTS-modulated radiofrequency exposure in an ethylnitrosourea mouse model", International Journal of Radiation Biology 86(7), pp. 529-541.

ZENI O., SANNINO A. et al. (2012), "Induction of an adaptive response in human blood lymphocytes exposed to radiofrequency fields: influence of the universal mobile telecommunication system (UMTS) signal and the specific absorption rate", Mutation Research 747(1), pp. 29-35. 